\title{
Use of strong opioids for chronic non-cancer pain: a retrospective analysis at a pain centre in Singapore
}

\author{
Jane Mary $\underline{\text { George }}^{1}$, MBBS, FAMS, Mahesh $\underline{\text { Menon }}^{1}$, MBBS, MD, Preeti Gupta ${ }^{1}$, MD, FRCA,
} Michelle Geoh Ean $\underline{T a n}^{1}$, MBBS, FFPMANZCA

INTRODUCTION The use of opioids in chronic non-cancer pain (CNCP) is controversial, as it presents both benefits and risks. There is currently no available data on the incidence, prescription pattern, functional outcomes and adverse effects of opioids in patients with CNCP in Singapore. This study aimed to address the aforementioned deficit. METHODS All records of patients who were prescribed strong opioids (for $>3$ months per year) for the management of CNCP over a two-year period were retrospectively analysed. Factors including type of opioid, indications for opioid prescription, uncontrolled side effects, functional status, coexisting psychological issues and suspicion of aberrant drug-seeking behaviour were studied.

RESULTS Out of the 1,389 new patients who visited the centre, 42 (3.0\%) with CNCP received strong opioids for more than three months a year. The most commonly prescribed opioid was methadone (42.9\%). The principal diagnosis for opioid prescription was spinal pain (38.1\%). Ten patients had severe side effects. 15 patients saw improvement in activities of daily living scores. Although ten patients returned to work, one stopped following the commencement of opioids. Aberrancy was seen in $5(11.9 \%)$ patients, while $19(45.2 \%)$ had psychological issues and $10(23.8 \%)$ required psychiatric co-management.

CONCLUSION Opioids are not a panacea for chronic pain. Therefore, functional outcomes should be considered more important end points than mere reductions in pain scores. A multidisciplinary team approach is essential for the effective management of patients with CNCP who are on opioids.

Keywords: Asia, chronic pain, non-cancer pain, opioids, Singapore

\section{INTRODUCTION}

Chronic pain is described as pain lasting for a duration of more than three months or persisting beyond the period of tissue healing. ${ }^{(1)}$ Chronic pain has a substantial impact on not only suffering individuals and their families, but also society in general. ${ }^{(2,3)}$ Traditionally, opioids have had a well-defined role in the management of cancer and acute pain. ${ }^{(4,5)}$ The effectiveness of opioids in cancer and acute pain management has prompted its use in chronic non-cancer pain (CNCP). Unfortunately, the liberal use of opioids in some countries has introduced a new set of problems, namely the misuse and diversion of opioids, dependence on opioids and death from overdose. ${ }^{(6-9)}$ This has prompted the publication of recommendations and guidelines by several international bodies, acknowledging the limited benefits of opioids in CNCP treatment and cautioning against the use of opioids due to its various associated complications. ${ }^{(10-14)}$

In a survey conducted in 2008, the incidence of chronic pain in Singapore was reported to be $8.7 \%,{ }^{(15)}$ with a majority of these patients suffering from CNCP. However, there is a paucity of data on the use of opioids in patients with CNCP in Asian countries, and no data from Singapore is currently available. This study, which aimed to address this deficit, was conducted to determine the incidence, prescription pattern, functional outcomes and adverse effects of opioid use in this group of patients in Singapore.

\section{METHODS}

Following institutional review board approval, all the records of patients seen at the Pain Management Centre, Singapore General Hospital, Singapore, over a two-year period (between January 2006 and March 2008), who were prescribed strong opioids for the control of CNCP for a duration of more than three months in a year, were retrospectively analysed. Information was gathered from written case records, direct communications with the pain physicians involved, pharmacy records and the hospital's electronic medical records, which are accessible to registered doctors at the hospital. Factors studied included type of opioids prescribed, indications for opioid prescription, uncontrolled side effects, and functional status with respect to the patient's ability to perform activities of daily living (ADL) and the ability to return to useful work. Coexisting psychological issues and aberrant drug-seeking behaviour while taking these strong opioids were also analysed. Pain scores were not determined prior to and after the commencement of opioids, as changes in functional status were thought to be more important outcomes than mere improvement of pain scores.

Strong opioids, for the purpose of this study, were defined as Class I drugs available in Singapore. These included morphine, methadone, oxycodone and fentanyl patch. Tramadol and codeine were excluded, as they are classified as weak opioids without significant dependence and abuse potential.

${ }^{1}$ Pain Management Centre, Singapore General Hospital, Singapore

Correspondence: Dr Michelle Tan, Senior Consultant Anaesthesiologist, Pain Management Centre, Singapore General Hospital, Outram Road, Singapore 169608. michelle.tan.g.e@sgh.com.sg 
ADL included standard activities such as eating, dressing, bathing, toileting and taking medication during the course of a normal day at the patient's place of residence. ${ }^{(16)}$ Baseline ADL function was recorded prior to the commencement of opioid therapy and reassessed after at least three months of opioid therapy. ADL function was considered 'improved' if a patient who did not previously have full ability to perform ADL became ADL-independent after opioid therapy. ADL function was considered 'not improved' if a patient was dependent in any ADL both before and after opioid therapy, or became ADL-dependent after opioid therapy. For a patient who had full ability to perform ADL before and after opioid therapy, ADL function was deemed to be 'unchanged'.

Engagement in work included not only employment outside the home, but also the ability to perform work as a homemaker or provide useful assistance within the home. Work status was considered to have 'improved' if patients who were previously not engaged in work were able to do so after opioid therapy, while it was considered 'not improved' if patients were not engaged in work before and after opioid therapy. For patients who were already working prior to taking opioids and continued to work while on opioids, the work status was classified as 'unchanged'.

Problematic drug use or aberrant drug-seeking behaviour was assessed and defined based on the criteria described by Chabal et $\mathrm{al}^{(17)}$ as follows: (a) persistent, overwhelming focus on opiate issues, which impedes progress with other issues regarding the patient's pain; (b) pattern of early refills $(\geq 3)$ or escalating drug use in the absence of an acute change in the patient's medical condition; (c) multiple telephone calls or visits to the administrative office to request for more opiates or early refills, or to inform about problems associated with the opiate prescription; (d) pattern of reporting prescription problems for a variety of reasons, including loss, spillage or misplacement of medications; and (e) supplemental sources of opiates obtained from multiple providers, emergency rooms or illegal sources. Coexistent psychological issues included the presence of life stressors (e.g. loss of a loved one, job loss), anxiety, family problems, emotional stress, and signs of depression (e.g. persistent low mood, sleep disturbance). Past and present psychiatric illnesses were also ascertained.

All statistical analyses were performed using the Statistical Package for the Social Sciences for Windows version 17 (SPSS Inc, Chicago, IL, USA).

\section{RESULTS}

A total of 42 non-cancer patients received strong opioids for more than three months in a year during the study period, accounting for $3.0 \%$ of new patients seen $(n=1,389)$. The mean age of the 42 patients was 48 (range 24-81) years, and women comprised $42.9 \%$ of the patients. Table I shows the details of the patients' opioid therapy. The most commonly used opioid was methadone $(45.2 \%)$, followed by morphine $(38.1 \%)$,
Table I. Details of opioid therapy in the patients $(n=42)$.

\begin{tabular}{lc}
\hline Variable & No. of patients (\%) \\
\hline Type of opioid* & \\
Methadone & $19(45.2)$ \\
Morphine & $16(38.1)$ \\
Oxycodone & $10(23.8)$ \\
Fentanyl patch & $4(9.5)$ \\
Morphine dose equivalent (mg) & \\
1 -30 & $15(35.7)$ \\
$31-60$ & $15(35.7)$ \\
$61-90$ & $4(9.5)$ \\
$91-120$ & $3(7.1)$ \\
$121-150$ & $1(2.4)$ \\
$\geq 151$ & $4(9.5)$ \\
Diagnosis & \\
Spine-related neuropathic pain & $15(35.7)$ \\
Nonspinal neuropathic pain & $8(19.0)$ \\
Chronic postsurgical pain & $5(11.9)$ \\
Visceral pain & $5(11.9)$ \\
Post-traumatic chronic pain & $2(4.8)$ \\
Headache & $2(4.8)$ \\
Other spine-related pain & $1(2.4)$ \\
Others & $4(9.5)$ \\
* Numbers add up to $>$ 42 patients, as two types of opioids were used for \\
some patients
\end{tabular}
some patients.

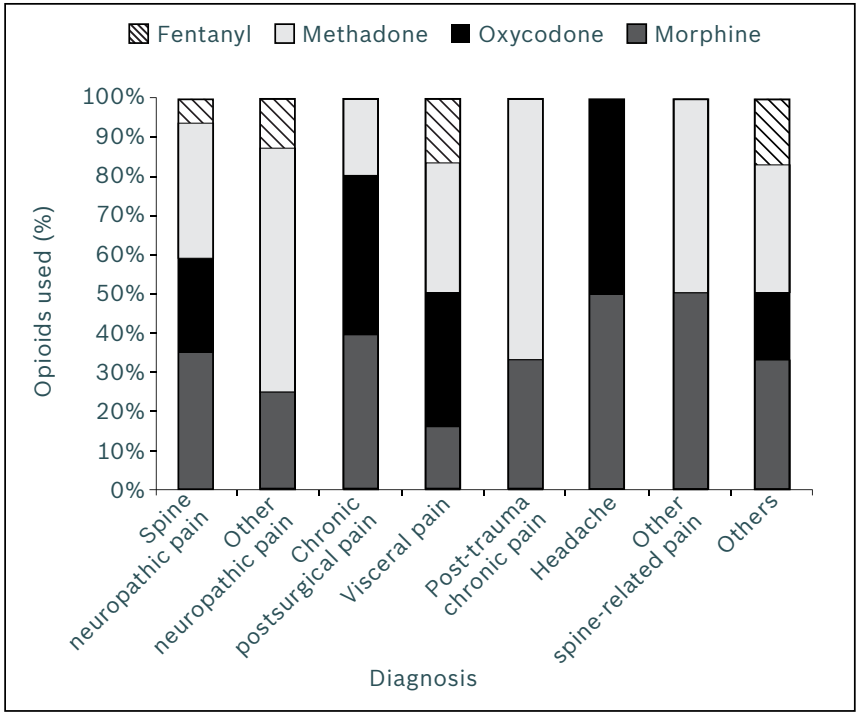

Fig. 1 Graph shows the distribution of opioids used in each diagnostic category.

oxycodone $(23.8 \%)$ and fentanyl patch $(9.5 \%)$. The mean dose of morphine (or its dose equivalent) was $65 \mathrm{mg}$, while its median dose was 45 (range 9.4-300) mg. The most common diagnosis for which opioids were prescribed was spine-related pain (neuropathic and nociceptive), which accounted for $38.1 \%$ of patients. Other diagnoses for which opioids were prescribed included nonspinal neuropathic pain (19.0\%), chronic postsurgical pain (11.9\%), visceral pain $(11.9 \%)$, headache $(4.8 \%)$, post-trauma chronic pain $(4.8 \%)$ and others $(9.5 \%)$. The four patients in the miscellaneous group had systemic sclerosis, ischaemic pain from an autoimmune disease, dermatitis artefacta and pain from a foreign body in the lung.

The distribution of opioids used within each diagnostic category is shown in Fig. 1. Morphine was used in all categories, while methadone was used in all but one category, i.e. patients with headaches. Dual opioid therapy was used in 
Table II. Functional outcome of opioid therapy in the patients $(n=42)$.

\begin{tabular}{lc}
\hline Variable & No. of patients (\%) \\
\hline Activities of daily living & \\
Improved & $15(35.7)$ \\
Not improved & $6(14.3)$ \\
Unchanged & $21(50.0)$ \\
Ability to work while on opioids* & \\
Improved & $10(23.8)$ \\
Not improved & $14(33.3)$ \\
Unchanged & $14(33.3)$ \\
Retired functionally & $2(4.8)$ \\
Stopped work & $1(2.4)$ \\
\hline${ }^{*}$ Data is missing for one patient.
\end{tabular}

${ }^{*}$ Data is missing for one patient.

seven patients and the remaining patients received only one type of opioid. Among the 42 patients who received strong opioids, 10 had severe side effects that could not be controlled with medication - constipation despite taking laxatives $(n=6)$, significant dizziness and nausea $(n=3)$, and urinary retention complicated by urinary tract infection $(n=1)$.

The outcomes of ADL assessment and the impact that opioids had on the patients' return to work are summarised in Table II. Improvement in ADL function was seen in 15 (35.7\%) patients who received opioid therapy, whereas $6(14.3 \%)$ failed to show any improvement. Half of the patients $(n=21)$ had an acceptable level of ability to perform ADL prior to opioid therapy. With regard to the ability to engage in work while on opioids, 14 (33.3\%) patients who were not working prior to opioid therapy did not return to work following treatment and $1(2.4 \%)$ patient stopped working after commencing opioids. A total of $14(33.3 \%)$ patients who were gainfully employed or working at home prior to opioid commencement continued to work after treatment, while $10(23.8 \%)$ patients who were previously not gainfully employed returned to work after starting opioid therapy.

In all, 19 (45.2\%) patients had clinically identifiable psychological issues and 10 (23.8\%) patients received formal intervention by a psychiatrist. Aberrancy was seen in 5 (11.9\%) patients - two patients had documented psychiatric disorders (bipolar), two had psychological issues (depression) and one had no psychological issues. Of the 42 patients who received opioid therapy, case records indicated that 30 had formal written opioid agreements with the prescribing physician and had provided informed consent. The remaining patients were informed verbally, with an intention to obtain a written agreement if the patient continued on opioids.

\section{DISCUSSION}

Our data revealed that about 3\% of all patients seen at our pain clinic were given strong opioids for a period of at least three months in a year. However, our cohort consisted of patients who had come to a tertiary hospital with severe pain, having previously exhausted other modalities of treatment. Direct comparison of our data with that of other countries is difficult, as most other studies have reported opioid use as a percentage of the population or in terms of the absolute dose of morphine taken over a period of time. Furthermore, our data was collected in a tertiary setting, whereas previous surveys on opioid prescription rates were taken from the general population. Nonetheless, in the United States, more than $3 \%$ of adults now receive long-term opioid therapy for $\mathrm{CNCP}^{(7)}$ Opioid prescription for musculoskeletal pain has doubled from $8 \%$ to $16 \%$ between 1980 and 2000. ${ }^{(18)}$ According to a study by Caudill-Slosberg et al, the use of more potent opioids has increased from $2 \%$ to $9 \%$ of visits for musculoskeletal pain complaints. ${ }^{(18)}$ In Denmark, an estimated $0.2 \%$ of the population was using opioids for chronic pain despite an estimated pain prevalence of $30 \%$, although this study also included patients with cancer. ${ }^{(19)}$ Bell reported that, in Australia, the amount of oral morphine consumed increased five-fold from $117 \mathrm{~kg}$ to $578 \mathrm{~kg}$ over a ten-year period from 1986 to $1996 .^{(20)}$

In our analysis, morphine and methadone were the most frequently prescribed opioids, accounting for about two-thirds of the total opioids used. Although oxycodone was officially approved for use in Singapore in 2005, its higher cost may have accounted for its lower usage in our cohort. In our study, dual opioid therapy was not commonly used for CNCP; it was mainly used for breakthrough pain and during conversion from one opioid to another.

Spine-related pain (38\%) was the principal reason why opioids were prescribed for our patients. This finding was similar to that of two studies from small primary care centres. ${ }^{(21,22)}$ One study was by Reid et al; ${ }^{(21)}$ the study, which involved patients with $\mathrm{CNCP}$ who received at least six months of opioid prescriptions, reported that low back pain (54\%) and spinal stenosis $(29 \%)$ accounted for most of the prescriptions. The other study was study by Adams et al; ${ }^{(22)}$ the study reported that spine-related pain or lower lumbar back pain (44\%), joint disease/arthritis (33\%) and headache/migraine pain (28\%) were the three most common reasons for opioid use. However, unlike our study, oxycodone was more frequently prescribed than morphine in these two studies.

Although intolerable side effects were seen in some of our patients, its incidence was surprisingly low. In our study, only $6(14.3 \%)$ patients had significant constipation despite using laxatives. Other studies have shown bowel-related dysfunction from opioids to be as high as $63.5 \%$, especially when morphine is used. ${ }^{(23)}$ The lower prevalence in our study may be dosedependent, but this association was not evaluated in detail. Also, we did not include patients who responded to simple laxatives. Despite medication, three patients reported significant dizziness and nausea, and one patient had opioid-induced urinary retention with urinary tract infection.

Established guidelines on the management of CNCP with opioids dictate that there should be demonstrable improvements in physical, psychological and social functions with opioid treatment. ${ }^{(10-12)}$ In a large Danish Health and 
Morbidity Survey, ${ }^{(24)}$ the authors analysed the results from a national random sample of 10,066 individuals, of whom 1,906 were identified as having CNCP. The study concluded that the use of opioids to manage CNCP did not fulfil opioid treatment goals, which included pain relief, improved quality of life and improved functional capacity. However, our study showed that about a quarter $(n=10)$ of patients were able to return to work after the initiation of opioids for pain control. Another 14 patients were able to continue working after the initiation of opioid therapy and two patients, although retired, were usefully occupied at home. Thus, a total of 26 (61.9\%) patients with $\mathrm{CNCP}$ on active opioid treatment in our study were able to function without becoming an economic burden. One-third of our patients did not return to useful work and one patient stopped working after initiating opioids, concurring with other reports that suggest that opioids do not always improve function. ${ }^{(13)}$

Patients who remain refractory to long-term opioid therapy for chronic pain may have psychiatric disorders and major coexistent psychological issues that act as barriers to effectiveness. ${ }^{(25)}$ About $30 \%-50 \%$ of patients presenting to chronic pain clinics meet the criteria for current major depression. ${ }^{(26)}$ In our analysis, 19 out of 42 (45.2\%) patients were identified as having coexistent psychological comorbidities, and $10(23.8 \%)$ patients received formal psychiatric treatment. According to the British Pain Society consensus statement on the use of opioids for $\mathrm{CNCP}^{(10)}$ the presence of a psychological comorbidity or a history of alcohol/problem drug use does not preclude the use of opioids. Provided patients comply with prescription instructions and have no suicidal potential, opioids do not necessarily have to be withheld. For such patients, it is more important to address any reversible psychosocial issues in a multidisciplinary framework while preventing addiction and maintaining function.

In our study, aberrancy was seen in 5 (11.9\%) patients. This result must be interpreted in the context of a study that was performed on a small sample visiting a tertiary centre. There is a paucity of literature on this subject from South Asia, and a study by Larance et al has noted that prescription medications abused in this region tend to be low-potency opioids. ${ }^{(27)}$ A review by Højsted and Sjøgren reported the prevalence of problematic drug use (including addiction, abuse, misuse and dependence) to be $0 \%-50 \% .{ }^{(28)}$ Such variable prevalence rates were probably due to differences in definitions, study design and population samples. According to a survey of primary care patients that adopted the Diagnostic and Statistical Manual of Mental Disorders, fifth edition (DSM-5) diagnostic criteria, the reported prevalence of opioid-use disorder was $34.9 \%$. ${ }^{(29)}$

The role of urinary drug testing (UDT) remains controversial. UDT is the most practical and objective tool available to prescribers for medically assessing, at any given point in time, whether a patient is taking prescribed medications or unauthorised controlled medications, or using illicit substances. ${ }^{(30)}$ However, UDT only provides a snapshot of the person's medication usage and cannot diagnose abuse, addiction or diversion. Such diagnoses can only be made after careful history taking, physical examination, acquisition of collateral information from family members, and the use of screening questionnaires.

Despite being recommended by pain societies overseas, there is only weak evidence that UDT can identify misuse, abuse, addiction and diversion. Other issues include prescribers having limited knowledge on UDT and not being trained to interpret UDT results. Only a small proportion of patients using opioids for CNCP abuse or divert their medications. Also, determining when to test patients, how often and what type of measures to use (laboratory versus point-of-care devices) has large cost implications for the patient, doctor and society. ${ }^{(31)}$ In Singapore, opioid prescriptions have a strong framework created to prevent misuse. ${ }^{(32,33)}$ In line with international guidelines and national policies pertaining to opioids, our Pain Management Centre has implemented departmental policies regarding the use of opioids so as to ensure that the best clinical practice and best patient outcome are achieved. The policies are listed below:

a. Ensure all pain management alternatives have been trialled, including physiotherapy, cognitive behavioural therapy, non-opioid analgesics and adjuvants.

b. Assess and address any mental health and/or substance abuse issues, with consideration of psychiatric or psychological referral.

c. Initiate a trial of opioids to ascertain improvement in function and/or pain, and monitor for any side effects.

d. Inform and counsel patients regarding the adverse effects of opioids, and responsible consumption and storage.

e. Ensure regular follow-up, and supply only the amount required till the subsequent follow-up date.

f. Ensure that an 'opioid agreement' has been initiated and signed. The opioid contract informs patients of possible side effects such as sedation, vomiting, tolerance and endocrine suppression. The patient has to agree that opioids or sedatives will not be obtained from other sources without notification and that he/she agrees to random drug sampling. This contract is terminated if the agreement is not adhered to or if the patient shows aberrant drug-seeking behaviour.

g. Ensure proper documentation of the 4 As at each visit Analgesia, Activity, Adverse effects and Abuse issues. Repeated re-evaluation is made after the initiation of therapy.

h. Ensure accurate and meticulous record keeping.

A balance has to be struck between the widespread prevalence of chronic pain - 19\% in Europe, ${ }^{(3)} 21 \%$ in the United Kingdom, ${ }^{(34)}$ approximately $30 \%$ in the United States ${ }^{(35)}$ and at least $8.7 \%$ in Singapore ${ }^{(15)}$ - and the use of opioids for the management of patients with chronic pain. Concerns regarding addiction, diversion, misuse and intolerable side effects 
remain significant barriers to the prescription of opioids. ${ }^{(36,37)}$ Other than the weak evidence supporting the use of opioids for $\mathrm{CNCP}^{(12)}$ the long-term outcomes of its use are also uncertain. ${ }^{(10)}$ However, recommendations by a panel of experts suggest that opioids can benefit a select group of patients with chronic pain, and if comprehensive risk assessment is performed, the benefits could outweigh potential harm. ${ }^{(14)}$

Drawbacks of this study include its retrospective nature and the short time frame used for data collection, which could have been extended to include more patients. Also, functional assessment would have been more complete if standardised questionnaires such as the Short Form Health Survey 36 had been used. It would be interesting, going forward, to collect data to determine whether prescribing practices and the spectrum of adverse outcomes have changed in Singapore and how many of these patients continue to be on opioids.

In conclusion, the opioid prescription rate for CNCP was $3.0 \%$ over a two-year period at our centre. We found that opioids helped improve the ability of about one-third of our patients to perform ADL and enabled a quarter of our patients to return to work. Uncontrollable side effects were seen in a quarter of patients on opioid therapy and aberrant drugseeking behaviour was present in $11.9 \%$ of patients. Our study highlights the importance of good patient selection, as opioids are not a panacea for CNCP. The goal of opioid therapy, when initiated, should be an improvement in the functional status of patients and not merely pain reduction. Strict opioid prescription guidelines need to be in place, and a multidisciplinary approach is essential to effectively manage patients with CNCP who are on opioids. Only those trained in managing this challenging group of patients should prescribe medication.

\section{ACKNOWLEDGEMENTS}

We are grateful to Ma Thin Mar Win and Stephanie Fook for their help with the statistics and figures.

\section{REFERENCES}

1. Turk DC, Okifuji A. Pain terms and taxonomies. In: Fishman SM, Ballantyne JC, Rathmell JP, eds. Bonica's management of pain, 4th ed. Philadelphia: Lippincott Williams \& Wilkins, 2010:13-4.

2. Phillips CJ, Harper C. The economics associated with persistent pain. Curr Opin Support Palliat Care 2011; 5:127-30.

3. Reid KJ, Harker J, Bala MM, et al. Epidemiology of chronic non-cancer pain in Europe: narrative review of prevalence, pain treatments and pain impact. Curr Med Res Opin 2011; 27:449-62.

4. Colson J, Koyyalagunta D, Falco FJ, Manchikanti L. A systematic review of observational studies on the effectiveness of opioid therapy for cancer pain. Pain Physician 2011; 14:E85-102.

5. Australian and New Zealand College of Anaesthetists and Faculty of Pain Medicine. Systemically administered analgesic drugs. In: Macintyre PE, Scott DA, Schug SA, eds. Acute pain management: the scientific evidence, 3rd ed. Melbourne: ANZCA, 2010: 57-64.

6. Paulozzi LJ , Budnitz DS, Xi Y. Increasing deaths from opioid analgesics in the United States. Pharmacoepidemiol Drug Saf 2006; 15:618-27.

7. Dunn KM, Saunders KW, Rutter CM, et al. Opioid prescriptions for chronic pain and overdose: a cohort study. Ann Intern Med 2010; 152:85-92.

8. Kuehn BM. Opioid prescriptions soar: increase in legitimate use as well as abuse. JAMA 2007; 297:249-51.

9. Paulozzi LJ, Ryan GW. Opioid analgesics and rates of fatal drug poisoning in the United States. Am J Prev Med 2006; 31:506-11.
10. The British Pain Society. Opioids for persistent pain: Good practice 2010. London: the British Pain Society, 2010: 36

11. Furlan AD, Reardon R, Weppler C; National Opioid Use Guideline Group. Opioids for chronic noncancer pain: a new Canadian practice guideline. CMAJ 2010; 182:923-30.

12. Chou R, Fanciullo GJ, Fine PG, et al. Clinical guidelines for the use of chronic opioid therapy in chronic noncancer pain. J Pain 2009; 10:113-30.

13. Manchikanti L, Ailinani $\mathrm{H}$, Koyyalagunta D, et al. A systematic review of randomized trials of long-term opioid management for chronic non-cancer pain. Pain Physician 2011;14:91-121.

14. Chou R, Ballantyne JC, Fanciullo GJ, Fine PG, Miaskowski C. Research gaps on use of opioids for chronic noncancer pain: findings from a review of the evidence for an American Pain Society and American Academy of Pain Medicine clinical practice guideline. J Pain 2009; 10:147-59.

15. Yeo SN, Tay KH. Pain Prevalence in Singapore. Ann Acad Med Singapore 2009; 38:937-42.

16. Covinsky K. Aging, arthritis, and disability. Arthritis Rheum 2006; 55:175-6.

17. Chabal C, Erjavec MK, Jacobson L, Mariano A, Chaney E. Prescription opiate abuse in chronic pain patients: clinical criteria, incidence and predictors. Clin J Pain 1997; 13:150-5.

18. Caudill-Slosberg MA, Schwartz LM, Woloshin S. Office visits and analgesic prescriptions for musculoskeletal pain in US: 1980 vs. 2000. Pain 2004; 109:514-9.

19. Sørensen HT, Rasmussen HH, Møller-Petersen JF, et al. Epidemiology of pain requiring strong analgesics outside hospital in a geographically defined population in Denmark. Dan Med Bull 1992; 39:464-7.

20. Bell JR. Australian trends in opioid prescribing for non-cancer pain, 1986-1996. Med J Aust 1997; 167:26-9.

21. Reid MC, Engles-Horton LL, Weber MB, et al. Use of Opioid Medications for Chronic Noncancer Pain Syndromes in Primary Care. J Gen Intern Med 2002; 17:173-9.

22. Adams NJ, Plane MJ, Fleming MF, et al. Opioids and the treatment of chronic pain in a primary care sample. J Pain Symptom Manage 2001; 22:791-6.

23. Rosti G, Gatti A, Costantini A, Sabato AF, Zucco F. Opioid-related bowel dysfunction: prevalence and identification of predictive factors in a large sample of Italian patients on chronic treatment. Eur Rev Med Pharmacol Sci 2010; 14:1045-50.

24. Eriksen J, Sjogren P, Bruera E, Ekholm O, Rasmussen NK. Critical issues on opioids in chronic non-cancer pain: an epidemiological study. Pain 2006; 125:172-9.

25. Clark MR, Galati SA. Opioids and psychological issues: A practical, patient-centered approach to a risk evaluation and mitigation strategy. Eur J Pain Suppl 2010; 4:261-7.

26. Dersh J, Polatin PB, Gatchel RJ. Chronic pain and psychopathology: research findings and theoretical considerations. Psychosom Med 2002; 64:773-86.

27. Larance B, Ambekar A, Azim T, et al. The availability, diversion and injection of pharmaceutical opioids in South Asia. Drug Alcohol Rev 2011; 30:246-54.

28. Højsted J, Sjøgren P. Addiction to opioids in chronic pain patients: a literature review. Eur J Pain 2007; 11:490-518.

29. Boscarino JA, Rukstalis MR, Hoffman SN, et al. Prevalence of prescription opioid-use disorder among chronic pain patients: comparison of the DSM-5 vs. DSM-4 diagnostic criteria. J Addict Dis 2011; 30:185-94.

30. Peppin JF, Passik SD, Couto JE, et al. Recommendations for urine drug monitoring as a component of opioid therapy in the treatment of chronic pain. Pain Med 2012; 13:886-96.

31. Schonwald G. What is the role of Urine Drug Testing (UDT) in the management of chronic non-cancer pain with opioids? Pain Med 2012;13:853-6.

32. Yee SK. What you need to know: guidelines to medical practitioners for proper maintenance of drugs and dispensing records (including controlled drugs). Singapore Med J 1998; 39:520-2.

33. AGC Singapore. Misuse of Drugs Act 2008. Chapter 185 [online]. Available at: statutes.agc.gov.sg. Accessed February 4, 2013.

34. British Pain Society. GfK NOP Pain Survey 2005. London: the British Pain Society publications, 2005.

35. Johannes CB, Le TK, Zhou X, Johnston JA, Dworkin RH. The prevalence of chronic pain in United States adults: results of an Internet-based survey. J Pain 2010; 11:1230-9.

36. Lin JJ, Alfandre D, Moore C. Physician attitudes toward opioid prescribing for patients with persistent noncancer pain. Clin J Pain 2007; 23:799-803.

37. Spitz A, Moore AA, Papaleontiou M, et al. Primary care providers' perspective on prescribing opioids to older adults with chronic non-cancer pain: a qualitative study. BMC Geriatr 2011; 11:35. 